\title{
Infrared Absorption in Acetanilide by Solitons
}

Careri, G.; Buontempo, U.; Carta, F.; Gratton, E.; Scott, Alwyn C.

Published in:

Physical Review Letters

Link to article, DOI:

10.1103/PhysRevLett.51.304

Publication date:

1983

Document Version

Publisher's PDF, also known as Version of record

Link back to DTU Orbit

Citation (APA):

Careri, G., Buontempo, U., Carta, F., Gratton, E., \& Scott, A. C. (1983). Infrared Absorption in Acetanilide by Solitons. Physical Review Letters, 51(4), 304-307. https://doi.org/10.1103/PhysRevLett.51.304

\section{General rights}

Copyright and moral rights for the publications made accessible in the public portal are retained by the authors and/or other copyright owners and it is a condition of accessing publications that users recognise and abide by the legal requirements associated with these rights.

- Users may download and print one copy of any publication from the public portal for the purpose of private study or research.

- You may not further distribute the material or use it for any profit-making activity or commercial gain

- You may freely distribute the URL identifying the publication in the public portal

If you believe that this document breaches copyright please contact us providing details, and we will remove access to the work immediately and investigate your claim. 


\title{
Infrared Absorption in Acetanilide by Solitons
}

\author{
G. Careri, U. Buontempo, and F. Carta \\ Istituto di Fisica, Università di Roma, I-00185 Roma, Italy \\ and \\ E. Gratton \\ Department of Physics, University of Illinois, Urbana, Illinois 61801 \\ and \\ A. C. $\operatorname{Scott}^{(a)}$ \\ Laboratory of Applied Mathematical Physics, The Technical University of Denmark, DK-2800 Lyngby, Denmark
} (Received 26 January 1983)

\begin{abstract}
The infrared spectrum of acetanilide shows a new band that is red shifted from the main amide-I maximum by about $15 \mathrm{~cm}^{-1}$, the intensity of which increases at low temperature. It is suggested that this band may arise from the creation of amide-I solitons that are similar (but not identical) to those proposed by Davydov for the alpha helix in proteins.
\end{abstract}

PACS numbers: $78.30 . \mathrm{Jw}$

In acetanilide $\left(\mathrm{CH}_{3} \mathrm{CONHC}_{6} \mathrm{H}_{5}\right)_{x}$, or $\mathrm{ACN}$, two close chains of hydrogen-bonded amide groups run through the crystal providing an interesting model for a one-dimensional array of hydrogen bonds, a system of obvious biological interest. ${ }^{1}$ The ir spectrum has been studied at room temperature, ${ }^{2}$ and the crystal structure has been determined. ${ }^{3}$ As previously briefly reported, ${ }^{1}$ we found, close to the conventional amide-I (essentially $\mathrm{C}=\mathrm{O}$ stretching) band, a new band of uncertain origin.

In this Letter we discuss the results of experiments that have been performed on $A C N$ and some ACN derivatives in several different physical preparations. Polycrystalline ACN samples were prepared in pellet form by finely mixing ACN microcrystals and ir grade $\mathrm{KBr}$. Amorphous ACN samples were prepared by vacuum deposition on Irtran II windows of evaporated ACN. The most relevant features of the ir experiments can be summarized as follows:

(1) On cooling of crystalline ACN samples, a new band appears at $1650 \mathrm{~cm}^{-1}$ (Fig. 1). No other major changes occur in the ir spectrum from 4000 to $800 \mathrm{~cm}^{-1}$. The intensity of this new band steadily increases from room temperature to $70 \mathrm{~K}$, then increases slowly down to $4 \mathrm{~K}$ (Fig. 2). The band at $1650 \mathrm{~cm}^{-1}$ is not present in amorphous samples but after annealing of the amorphous material it is fully recovered.

(2) We assigned the new band at $1650 \mathrm{~cm}^{-1}$ to an amide-I component on the following bases: (i) ${ }^{15} \mathrm{~N}$ substitution which induces a small shift on the amide-I at $1665 \mathrm{~cm}^{-1}$ also shifts the new band by the same amount (Fig. 3). (ii) Deuterium substitution at the $\mathrm{NH}$ position strongly affects both the amide $-I$ and the new band in a complex way that will be reported at a later time. (iii) Upon cooling a parallel decrease of the normal amide $-I$ integrated absorption and increase of the 1650 band integrated absorption is observed. (iv) The 1650 band and the amide-I band show the same dichroism over the temperature range investigated.

(3) Several other experiments have been performed in order to clarify the nature of the 1650

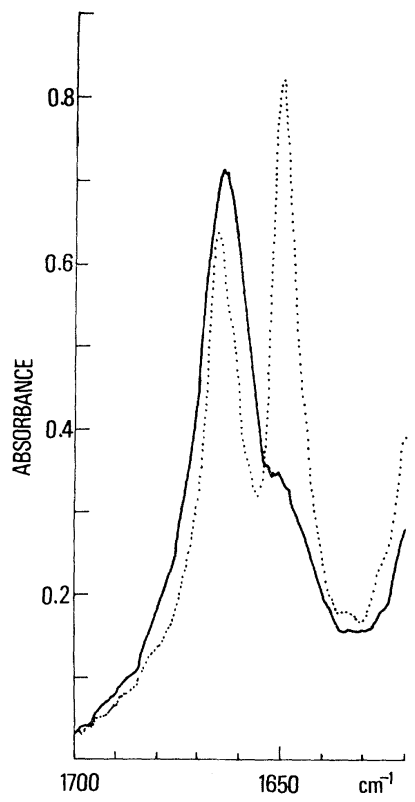

FIG. 1. ir spectra of crystalline ACN in the amide-I region (solid line, $300 \mathrm{~K}$; dotted line, $80 \mathrm{~K}$ ). 


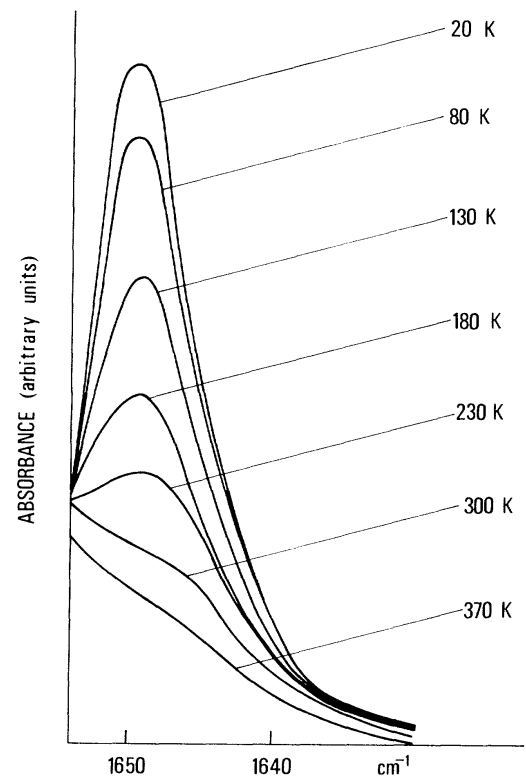

FIG 2. Detailed temperature dependence of the 1650$\mathrm{cm}^{-1}$ band.

band. (i) The hydrogen-bonded network of ACN crystals is essential for the appearance of the 1650 band. In fact ACN derivatives (such as ACN methylated at the position where the hydrogenbonded distances occur) do not show the 1650 band of ACN. (ii) Raman spectra of ACN show this 1650 band with the same temperature dependence as in the ir. (iii) A parallel study of temperature dependence has been carried out on the specific heat, the dielectric constant, and the volume expansion as detected by $\mathrm{x}$ rays in order to rule out the occurrence of rotational isomerism or of a polymorphic transition. Such transitions would affect some other ir and Raman absorption bands, a fact which has not been observed. Details of this experimental work will be reported elsewhere.

We summarize this brief survey of experimental results by emphasizing that the new band at $1650 \mathrm{~cm}^{-1}$ is characteristic of the amide-I group of ACN in crystal form. Recent calculations of amide modes in $\mathrm{ACN}$ do not show this band. ${ }^{4}$ Having excluded conventional explanations, we consider here the possibility of assigning it to a collective excitation similar to the soliton proposed by Davydov for the $\alpha$ helix in proteins.,

Davydov's soliton arises from a cooperative interaction between localized amide-I bond energy and lattice distortion. The bond energy acts, through a nonlinear coupling, as a source of lattice distortion. This lattice distortion reacts,

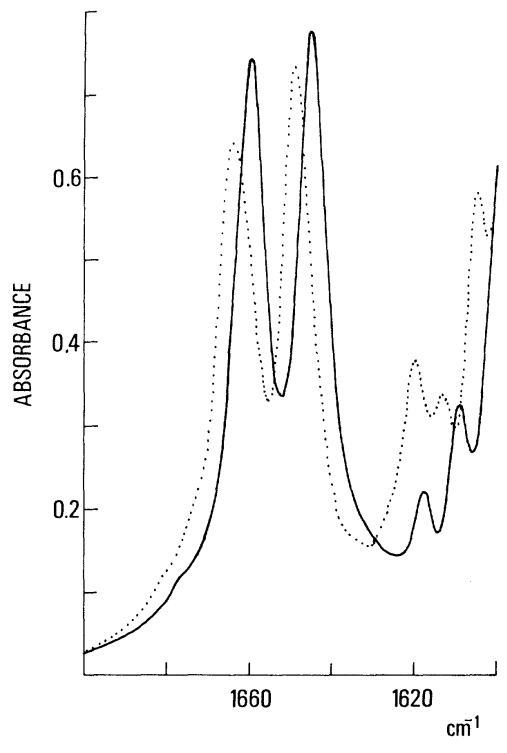

FIG. 3. ir spectra of crystalline normal ACN (dotted line) and ${ }^{15} \mathrm{~N}$-substituted $\mathrm{ACN}$ (solid line) at $80 \mathrm{~K}$.

again through the nonlinear coupling, as a potential well to trap the bond energy and prevent its dispersion via dipole-dipole coupling effects. We follow the same scheme with one important difference: For lattice distortion we substitute displacement of the hydrogen-bonded proton. Following Refs. 5-7, the probability amplitudes for amide-I vibrational quanta along the first chain $\left\{a_{n 1}\right\}$ are governed by the Schrödinger equations

$$
\begin{aligned}
& i \hbar \dot{a}_{n 1} \\
& =\left(E_{0}+\chi y_{n 1}\right) a_{n 1}-J\left(a_{n+1,1}+a_{n-1,1}\right)+L a_{n 2},
\end{aligned}
$$

where $n$ counts amide-I bonds along the chain, $E_{0}$ is the amide-I vibrational energy, $y_{n 1}$ is the average displacement of a hydrogen-bonded proton essentially along its $\mathrm{NH}$ bending, ${ }^{2} J$ is a longitudinal dipole-dipole interaction, $L$ is an interchain dipole-dipole interaction term, and $\chi$ is a nonlinear coupling that accounts for the change in amide-I vibrational energy per unit displacement of the hydrogen-bonded proton. On a time scale that is short compared with lattice motion we couple to (1) the average equations for motion of the hydrogen-bonded protons,

$$
\ddot{y}_{n 1}+\omega_{L}^{2} y_{n 1}=-\left(\chi / m_{p}\right)\left|a_{n 1}\right|^{2},
$$

where $m_{p}$ is proton mass and $\omega_{D}$ is the frequency of the $\mathrm{NH}$ deformation mode. We are primarily interested in a time-independent solution for which $\dot{y}_{n 1}=0$. Then (1) and (2) can be combined 
to give

$$
i \hbar \dot{a}_{n 1}-\left(E_{0}-2 J\right) a_{n 1}+J\left(a_{n+1,1}-2 a_{n 1}+a_{n-1,1}\right)+\left(\chi^{2} / m_{p} \omega_{L}^{2}\right)\left|a_{n 1}\right|^{2} a_{n 1}=L a_{n 2} \cdot
$$

Approximating (3) by a partial differential equation in which the integers $n$ are replaced by a continuous spatial variable $\xi=\boldsymbol{n} b$ (where $b$ is the longitudinal distance between amide-I bonds), scaling the space variable by $\xi=b(J / \hbar)^{1 / 2} x$, and scaling amplitudes by $a_{r}=(\hbar / J)^{1 / 4} A_{r}$ puts the dynamic equations into the form of the soliton equations, ${ }^{8}$

$$
\begin{aligned}
& i \dot{A}_{1}-\hbar^{-1}\left(E_{0}-2 J\right) A_{1}+A_{1, x x}+\alpha\left|A_{1}\right|^{2} A_{1} \cong \hbar^{-1} L A_{2}, \\
& i \dot{A}_{2}-\hbar^{-1}\left(E_{0}-2 J\right) A_{2}+A_{2, x x}+\alpha\left|A_{2}\right|^{2} A_{2} \cong \hbar^{-1} L A_{1},
\end{aligned}
$$

where $\alpha \equiv \chi^{2} / m_{p} \omega_{L}^{2}(\hbar J)^{1 / 2} \mathrm{sec}^{-1 / 2}$. Equations (4) have the energy functional

$$
\mathfrak{H C}=\left(E_{0}-2 J\right)\left(\left|A_{1}\right|^{2}+\left|A_{2}\right|^{2}\right)+L\left(A_{1} * A_{2}+A_{1} A_{2}^{*}\right)+\hbar\left[\left(\left|A_{1, x}\right|^{2}+\left|A_{2, x}\right|^{2}\right)-\frac{1}{2} \alpha\left(\left|A_{1}\right|^{4}+\left|A_{2}\right|^{4}\right)\right] .
$$

To interpret the spectra shown in Fig. 1 we consider three ir-active solutions of (4) that satisfy the single-quantum condition $\int\left(\left|A_{1}\right|^{2}+\left|A_{2}\right|^{2}\right) d x=1$ :

(1) Symmetric exciton at $k=0$. In this case $A_{1}=A_{2}=A_{0} \exp \left[-i t\left(E_{0}-2 J+L\right) / \hbar\right]$ where $A_{0}{ }^{-2}$ equals the number of amide-I bonds in the crystal. From (5) the exciton energy is $E_{1}=E_{0}-2 J+L$.

(2) Symmetric soliton on two chains. Again we take

$$
A_{1}=A_{2}=(\alpha / 32)^{1 / 2} \operatorname{sech}(\alpha x / 8) \exp \left\{-i t\left[\left(E_{0}-2 J+L\right) / \hbar-\alpha^{2} / 64\right]\right\},
$$

and the energy in joules is

$$
E_{2}=E_{0}-2 J+L-\chi^{4} /\left(192 \omega_{D}^{4} m_{p}^{2} J\right) \text {. }
$$

(3) Soliton on a single chain. In this case $A_{2}=0$ and

$$
A_{1}=(\alpha / 8)^{1 / 2} \operatorname{sech}(\alpha x / 4) \exp \left\{-i t\left[\left(E_{0}-2 J\right) / \hbar-\alpha^{2} / 16\right]\right\}
$$

so that the energy is $E_{3}=E_{0}-2 J-\chi^{4} / 48 \omega_{D}^{4} m_{p}{ }^{2} J$.

To obtain numerical values for these expressions we calculate the dipole-dipole interaction energies in the usual approximation ${ }^{4,9,10}$ as $J$ $=5.8 \mathrm{~cm}^{-1}$ and $L=1.7 \mathrm{~cm}^{-1}$, and we assume $\omega_{L}$ to be the frequency of the amide $-\mathrm{V}$ mode ( $\mathrm{CN}$ torsion and $\mathrm{NH}$ out-of-plane bending) at 700 $\mathrm{cm}^{-1} \cdot 9,11$

Taking $E_{1}=1665 \mathrm{~cm}^{-1}$ and supposing that the new peak may arise either from absorption by a symmetric soliton $\left(E_{2}=1650 \mathrm{~cm}^{-1}\right)$ or by a singlechain soliton $\left(E_{3}=1650 \mathrm{~cm}^{-1}\right)$, we obtain $\chi=2.7$ $\times 10^{-10} \mathrm{~N}$ and $1.9 \times 10^{-10} \mathrm{~N}$, respectively. From analysis of the experimental amide-I frequencies and the corresponding hydrogen bond lengths, we obtain a value for the change in amide-I vibrational energy per unit of longitudinal stretching of the adjacent hydrogen bond in the range (1.01.4) $\times 10^{-10} \mathrm{~N}$. A recent self-consistent-field calculation of this parameter indicates a value in the range $0.3-0.5 \mathrm{~N}^{12}$ The numerical results should be considered only as the best currently available, noting several sources of uncertainty: (i) Our treatment accounts only for nearestneighbor dipole interactions. (ii) In calculating dipole interaction energies we have assumed the dielectric permittivity of the vacuum. (iii) We calculate energies from (5) which depends upon a continuum approximation to (3). (iv) We compare evaluations of the change of amide-I energy per unit of out-of-plane proton displacement $(\chi)$ with calculations of the change per unit of longitudinal displacement.

Davydov $^{6}$ suggests that soliton excitation directly by light will be small for two reasons. First the radiation lifetime for excitons, $\tau_{\mathrm{ex}}$, is related to the radiation lifetime of an isolated amide-I bond, $\tau_{0}$, by $\tau_{\text {ex }} \cong(b / \lambda) \tau_{0}$ where $b$ is the distance between amide-I bonds. This implies that the corresponding absorption cross sections stand in the ratio $\sigma_{0} / \sigma_{\mathrm{ex}} \cong b / \lambda \ll 1$. Although true, these facts do not influence the results of an absorption experiment because the number of ir-active exitons per unit distance in the beam direction is $b / \lambda$ times the corresponding number of amide-I bonds. Thus the total absorption from the beam will be the same whether one considers it to be caused by excitons or by individual amide-I bonds. Davydov's second point about direct soliton absorption of light is that there is not sufficient time for the necessary lattice distortion to take place while the photon is being absorbed (Franck-Condon principle). In 
our case this does not apply because we do not require lattice distortion to form a soliton but only displacement of the ground state of the hydrogen-bonded proton. From (2) this can occur in a time of order $\omega_{D}^{-1}$ which is close to a period of amide-I oscillation. We expect the low-temperature intensity of the soliton line to be larger than that of the amide-I line because a soliton is spread over several amide-I bonds, and this is seen in Fig. 1.

To estimate the temperature dependence of the maximum absorbance of the $1650-\mathrm{cm}^{-1}$ line (Fig. 2 ), we consider thermal fluctuations of the proton about its mean position. Equation (1) shows that such fluctuations detune the soliton from the incident beam and reduce absorbance. If we assume that a proton is raised slightly above its ground state to $|\psi\rangle=a|0\rangle+b|1\rangle$ where $a=\left[1-\exp \left(-\hbar \omega_{D} / k T\right)\right]^{1 / 2}$ and

$$
b=\exp \left(-\hbar \omega_{L} / 2 k T\right)\left[1-\exp \left(-\hbar \omega_{L} / k T\right)\right]^{1 / 2},
$$

its average position (dropping subscripts) is $\langle\psi|\hat{y}| \psi\rangle=y+y_{T}(t)$ where

$$
y_{T}=\left(\frac{2 \hbar}{m_{p} \omega_{D}}\right)^{1 / 2} \exp \left(-\frac{\hbar \omega_{D}}{2 k T}\right)\left[1-\exp \left(-\frac{\hbar \omega_{D}}{k T}\right)\right] \cos \omega_{D} t .
$$

Replacing $y$ by $y+y_{T}$ in (1) we calculate the thermal detuning as $\omega=\omega_{\max } \cos \omega_{L} t$ where

$$
\omega_{\max } \equiv \chi\left(\frac{2}{m_{p} \omega_{D} \hbar}\right)^{1 / 2} \exp \left(-\frac{\hbar \omega_{D}}{2 k T}\right)\left[1-\exp \left(-\frac{\hbar \omega_{D}}{k T}\right)\right] .
$$

Assuming the probability of detuning to be $\left(2 \omega_{\max }\right)^{-1}$ for $|\omega|<\omega_{\max }$ and zero otherwise, and approximating the low-temperature shape of the $1650-\mathrm{cm}^{-1}$ line by a Lorentzian function of half width $\Delta \omega$, we calculate the relative maximum absorbance

$$
A(T) \equiv \omega_{\max }{ }^{-1} \int_{0}^{\omega \max }\left[1+(2 \omega / \Delta \omega)^{2}\right]^{-1} d \omega
$$

to be

$$
A(T)=\frac{\Delta \omega}{2 \omega_{\max }} \tan ^{-1}\left(\frac{2 \omega_{\max }}{\Delta \omega}\right) .
$$

In Fig. 4 we plot (6) for $\omega_{D}=700 \mathrm{~cm}^{-1}, \Delta \omega$ $=7.1 \mathrm{~cm}^{-1}$, and representative values of $\chi$. Best agreement with measured values is obtained for $x \approx 2 \times 10^{-10} \mathrm{~N}$.

In conclusion we remark that we have confirmed the $1650-\mathrm{cm}^{-1}$ band shown in Fig. 1 in many experiments over the past decade, but these meas urements were not published because a theoretical assignment was not available. Our soliton model seems to provide an explanation for this persistent experimental fact.

This work was supported in part by a grant from the Consiglio Nazionale delle Ricerche, Italy, and by Laboratori Ricerche di Base, SNAMProgetti at Monterotondo. (a) On leave from Los Alamos National Laboratory, Los Alamos, N. Mex. 87544.

${ }^{1} \mathrm{G}$. Careri, in Cooperative Phenomena, edited by H. Haken and M. Wagner (Springer, Berlin, 1973), p. 391.

${ }^{2}$ N. B. Abbott and A. Elliott, Proc. Roy. Soc. London, Ser. A 234, 247 (1956).

${ }^{3}$ C. J. Brown and D. E. C. Corbridge, Acta Crystallogr. 7,711 (1954); C. J. Brown, Acta Crystallogr. 21, 442 (1966).

${ }^{4} \mathrm{Yu}$. N. Chirgadze and N. A. Nevskaya, Biopolymers 15, 607 (1976).

${ }^{5}$ A. S. Davydov and N. I. Kislukha, Phys. Status Solidi (b) $\underline{59}, 465$ (1973). See A. S. Davydov, Phys. Scr. 20, 387 (1979) for an extensive bibliography.

${ }^{6}$ A. S. Davydov, Biology and Quantum Mechanics (Pergamon, New York, 1982).

${ }^{7}$ A. C. Scott, Phys. Rev. A 26, 578 (1982).

${ }^{8}$ A. C. Scott, Phys. Scr. 25, 651 (1982).

${ }^{9}$ W. H. Moore and S. Krimm, Biopolymers 15,2439 (1976).

${ }^{10} \mathrm{Yu}$. N. Chirgadze and Ye. P. Rashevskaya, Biophysics 14, 642 (1969), and Biofizika 14, 608 (1969).

${ }^{11}$ H. K. Kessler and G. B. B. M. Sutherland, J. Chem. Phys. 21, 570 (1953).

${ }^{12}$ V. A. Kuprievich and Z. G. Kudritskaya, Institute for Theoretical Physics, Kiev, Reports No. ITP-82-62E, No. ITP-82-63E, and No. ITP-82-64E (to be published). 